\title{
miR-1184 regulates inflammatory responses and cell apoptosis by targeting TRADD in an LPS-induced cell model of sepsis
}

\author{
PING LING, RONG TANG, HUAZHU WANG, XIUQIN DENG and JIANLI CHEN \\ Pediatric Intensive Care Unit, Guiyang Maternal and Child Health Care Hospital, Guiyang, Guizhou 550003, P.R. China
}

Received May 27, 2020; Accepted September 11, 2020

DOI: $10.3892 / \mathrm{etm} .2021 .10062$

\begin{abstract}
MicroR NAs (miRs) have been reported to be potential clinical biomarkers for sepsis. miR-1184 is a multifunctional microRNA that exerts roles in the development of various diseases. However, the role of miR-1184 in children with sepsis remain unknown. In the present study, THP-1 cells were stimulated with $1 \mu \mathrm{g} / \mathrm{ml}$ lipopolysaccharide (LPS) for $24 \mathrm{~h}$ to establish an in vitro sepsis model. Reverse transcription-quantitative PCR was used to evaluate the expression of miR-1184 in clinical specimens, and of IL-6, TNF- $\alpha$, IL-1 $\beta$, miR-1184 and TNF receptor type 1-associated DEATH domain protein (TRADD) in cells with and without LPS treatment. Cell apoptosis was assessed using flow cytometry. Binding between miR-1184 and TRADD was predicted using bioinformatics software, and a luciferase reporter assay was performed to verify the interaction between miR-1184 and TRADD in LPS-induced THP-1 cells. In addition, western blot analysis was performed to detect TRADD and proteins associated with the NF- $\kappa$ B pathway. The results showed that miR-1184 was downregulated in the blood of children with sepsis and LPS-induced THP-1 cells. Overexpression of miR-1184 alleviated the LPS-induced production of inflammatory cytokines and cell apoptosis. Moreover, TRADD was verified to be a direct target of miR-1184. Upregulation of TRADD reversed the effects of miR-1184 on the LPS-induced inflammatory response and apoptosis of THP-1 cells. Furthermore, the $\mathrm{NF}-\kappa \mathrm{B}$ pathway was shown to be associated with the regulatory role of miR-1184 in sepsis. The present study provides evidence that miR-1184 exerts inhibitory effects on inflammatory responses and apoptosis in sepsis by targeting TRADD, which suggests that miR-1184 may be a novel potential target for the therapy of children with sepsis.
\end{abstract}

Correspondence to: Dr Jianli Chen, Pediatric Intensive Care Unit, Guiyang Maternal and Child Health Care Hospital, 63 Ruijin South Road, Nanming, Guiyang, Guizhou 550003, P.R. China

E-mail: jianlichen_gysfybj@163.com

Key words: sepsis, microRNA-1184, TNF receptor type 1-associated DEATH domain protein, inflammation, apoptosis

\section{Introduction}

Sepsis is an excessive systemic inflammatory reaction caused by infection or trauma and accompanied by organ dysfunction and respiratory failure (1-3). Sepsis is one of the main causes of death of critically ill patients in intensive care units due to the lack of specific diagnostic markers and broad-spectrum therapies that are restricted to antibiotics and supportive care $(4,5)$. Currently, sepsis is a leading cause of morbidity and mortality in children worldwide (6). Due to the non-specific clinical characteristics of sepsis, the difficulty in differentiating sepsis from non-infectious etiologies and the low positivity rates of blood culture, the early diagnosis and treatment of children with sepsis is complicated and challenging $(7,8)$. Thus, it is urgently necessary to discover novel and accurate biomarkers and therapeutic targets for sepsis at an earlier stage.

MicroRNAs (miRs/miRNAs) are small, single-stranded endogenous RNA molecules that bind to target mRNAs to regulate gene expression and induce translational repression in eukaryotes, thereby serving vital roles in complicated human diseases (9-11). There is evidence that aberrant miRNA expression is associated with various autoimmune and inflammatory diseases, including sepsis (12). For example, in one study, miR-15a and miR-16 were reported to be upregulated in neonatal patients with sepsis, and to suppress lipopolysaccharide (LPS)-induced toll-like receptor (TLR)-4/interleukin (IL)-1 receptor-associated kinase 1 signaling in vitro, thereby inhibiting LPS-induced inflammation (13). In another study, it was observed that miR-375 levels were downregulated and miR-21 levels were upregulated in patients with sepsis, and a negative correlation was detected between the expression of these two miRNAs. Furthermore, the ectopic expression of miR-375 reduced the number of marrow-derived suppressor cells in a mouse model of sepsis, thus inhibiting sepsis development (14). Another miRNA, miR-1184, has been shown to be significantly decreased in the blood of neonates with sepsis (15). However, the role of miR-1184 and its underlying mechanism in children with sepsis remain to be elucidated.

The present study detected the downregulation of miR-1184 expression in the blood of children with sepsis and analyzed the effects of miR-1184 on inflammation and apoptosis in an LPS-induced cell model of sepsis. In addition, the present study investigated the target gene regulated by miR-1184 in order to further understand the molecular mechanism of sepsis. 


\section{Materials and methods}

Clinical specimens. The peripheral blood samples of 30 children (13 female, 17 male) with sepsis and 30 healthy control children (13 female, 17 male) were obtained at the Guiyang Maternal and Child Health Care Hospital between January 2017 and March 2019. The age range of control group was 8 to 11 years and the age range of sepsis group was 8 to 12 years. The length of hospitalization of control group was $42.9 \pm 10.3 \mathrm{~h}$ and the length of hospitalization of sepsis group was 43.9 \pm 11.9 . Experiments were approved by the Ethics Committee of Guiyang Maternal and Child Health Care Hospital and written informed consent was signed by the parents or guardians of all participants. Inclusion criteria: i) complete clinical data and ii) consent was obtained from the parents or guardians of the child and informed consent was signed. Exclusion criteria: i) children discharged from hospital within $24 \mathrm{~h}$ after admission; ii) onset time exceeds $72 \mathrm{~h}$; iii) children with any other health conditions complicated with major visceral lesions such as those affecting the heart, liver and/or kidney; iv) withdrawal from the study.

For the collection of peripheral blood serum, blood samples were centrifuged at $400 \mathrm{x}$ g for $10 \mathrm{~min}$ at $4^{\circ} \mathrm{C}$. The supernatant was collected immediately and stored at $-80^{\circ} \mathrm{C}$ prior to further analysis. For the collection of peripheral blood mononuclear cells, blood samples with anti-coagulation treatment (blood was stored in anticoagulant tubes, which contains sodium heparin) were added to an equal volume of lymphocyte isolation buffer (Beijing Solarbio Science \& Technology Co., Ltd.), and centrifuged at $800 \times \mathrm{g}$ for $18 \mathrm{~min}$ at $20^{\circ} \mathrm{C}$. The upper white layer was slowly transferred into a new tube with $10 \mathrm{ml}$ serum-free RPMI-1640 medium (Sigma-Aldrich; Merck KGaA), followed by centrifugation at $600 \mathrm{x} \mathrm{g}$ for $15 \mathrm{~min}$ at $20^{\circ} \mathrm{C}$. Following removal of the supernatant, cell precipitates were resuspended in RPMI-1640 medium and centrifuged at $400 \mathrm{x} \mathrm{g}$ for $8 \mathrm{~min}$ at $20^{\circ} \mathrm{C}$. The collected cells were mononuclear cells.

Cell culture and treatment. The THP-1 human monocytic cell line was obtained from The Cell Bank of Type Culture Collection of the Chinese Academy of Sciences and maintained in RPMI-1640 medium supplemented with 10\% FBS (HyClone; Cytiva), $100 \mu \mathrm{g} / \mathrm{ml}$ streptomycin and $100 \mathrm{U} / \mathrm{ml}$ penicillin at $37^{\circ} \mathrm{C}$ in a humidified incubator with $5 \% \mathrm{CO}_{2}$. To establish the in vitro sepsis model, THP-1 cells were treated with $1 \mu \mathrm{g} / \mathrm{ml}$ LPS (Sigma-Aldrich; Merck KGaA) for $24 \mathrm{~h}$ to simulate a septic environment at $37^{\circ} \mathrm{C}(16,17)$.

Cell Counting Kit 8 (CCK8). Cell proliferation was assessed using CCK-8 (Dojindo Molecular Technologies, Inc.) according to the instructions of the manufacturer. Cells were seeded at a density of 8,000 cells/well in a plate with 96 wells in complete medium overnight. The cells were treated accordingly a in $5 \% \mathrm{CO}_{2}$ at $37^{\circ} \mathrm{C}$ for $72 \mathrm{~h}$. A $10 \mu \mathrm{l}$ volume of CCK-8 solution was added to each well and the absorbance at $450 \mathrm{~nm}$ in $4 \mathrm{~h}$ was measured using a microplate reader (Thermo Fisher Scientific, Inc.).

Cell transfection. Cells were inoculated in a six-well plate at a concentration of $1 \times 10^{5} /$ well, and transfection experiments were conducted after the cells grew to $70-80 \%$ confluence. A miR-1184-overexpression plasmid (miR-1184 double-stranded mimic: 5'-AUUUCCCGCGGUUUCAAACUCUCGGC-3', forward; 5'-UAAACGCGCUUCAACGCCUGCGUUAAA-3', reverse), TRADD overexpression vector (pcDNA-TRADD) and corresponding negative controls (miR-NC and pcDNA-NC) were designed and synthesized by Shanghai GenePharma Co., Ltd. The vectors or negative controls were transfected at a concentration of $20 \mathrm{nM}$ into THP-1 cells using Lipofectamine $^{\mathrm{TM}} 2000$ (cat. no. 11668027; Invitrogen; Thermo Fisher Scientific, Inc.) in accordance with the manufacturer's instructions. Follow-up experiments were conducted $48 \mathrm{~h}$ after transfection.

Reverse transcription-quantitative PCR (RT-qPCR). Cells were inoculated in a $96-$ well plate at a concentration of $1 \times 10^{3} /$ well. The cells were treated with or without LPS accordingly and then total RNA was extracted from THP-1 cells and blood specimens using TRIzol ${ }^{\circledR}$ reagent (Invitrogen; Thermo Fisher Scientific, Inc.) according to the manufacturer's instructions. The extracted RNA was reverse transcribed into cDNA using the PrimeScript ${ }^{\mathrm{TM}}$ RT reagent kit (Takara Bio, Inc.) at about $65^{\circ} \mathrm{C}$ for $10 \mathrm{~min}$. cDNA amplification was performed via qPCR using the SYBR Premix Ex Taq ${ }^{\mathrm{TM}}$ II kit (Takara Bio, Inc.) on an ABI PRISM 7900 Real-Time system (Applied Biosystems; Thermo Fisher Scientific, Inc). Amplification conditions were as follows: $95^{\circ} \mathrm{C}$ for $10 \mathrm{~min}$, followed by 40 cycles of $95^{\circ} \mathrm{C}$ for $10 \mathrm{sec}$ and $60^{\circ} \mathrm{C}$ for $60 \mathrm{sec}$. GAPDH and U6 served as the internal controls. The primer sequences were as follows, IL-6 forward, 5'-GGCCCTTGCTTTCTCTTCG-3' and reverse, 5'-ATAATAAAGTTTTGATTATGT-3'; TNF- $\alpha$ forward, 5'-CAGCСТСТTCTCСТTCСТGA-3' and reverse, 5'-GGAAGACCCCTCCCAGATAGA-3'; IL-1 $\beta$ forward, 5'-GGCGAATTCCTTCAT TGCCCAGGT TTC-3' and reverse, 5'-GGCGAATTCCTTCATTGCCCAGGTTTC-3'; GAPDH forward, 5'-TGGGTGTGAACCACGAGAA-3' and reverse, 5'-GGCATGGACTGTGGTCATGA-3'; U6 forward 5'-CTCGCTTCGGCAGCACA-3' and reverse, 5'-ACGCTTCACGAATTTGC-3'. The relative expression levels of target genes were calculated using the $2^{-\Delta \Delta \mathrm{Cq}}$ method (18). Agarose gel electrophoresis (1\%) was used to detect the PCR products. In brief, $8 \mu \mathrm{l}$ PCR products and $2 \mu \mathrm{l}$ sample loading buffer were mixed for sample loading and a DNA Marker of appropriate size was used for electrophoresis separation with ethidium bromide staining. The results were analyzed by ImageQuant TL (version 7.0, GE Healthcare; Cytiva).

Cell apoptosis analysis. The cells were grown at a density of $1 \times 10^{5}$ in a six-well plate. Flow cytometry was performed to detect apoptosis of the cells using an FITC Annexin V/PI Apoptosis Detection kit I (Guangzhou RiboBio Co., Ltd.). Cells with or without prior LPS treatment were transfected with miR-1184 mimic or miR-NC, alone or in combination with pcDNA-NC or pcDNA-TRADD for $48 \mathrm{~h}$. Subsequently, cells were collected, resuspended and stained with Annexin V/PI reagent according to the manufacturer's instructions. FlowJo software (version 7.6.1; FlowJo LLC.) was used to analyze cells in the early and late stages of apoptosis. Each experiment was repeated three times. 
A

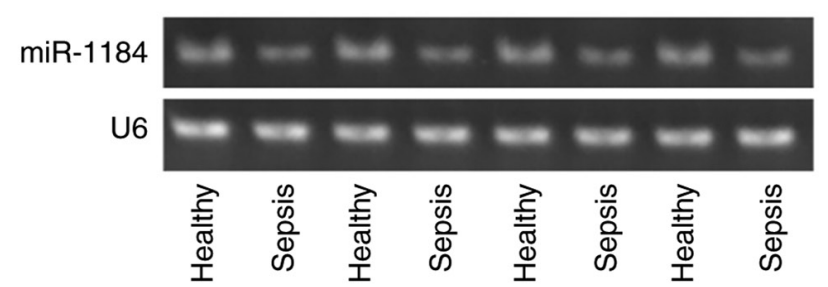

B

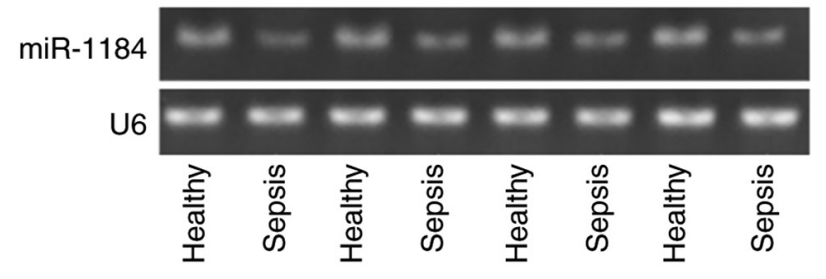

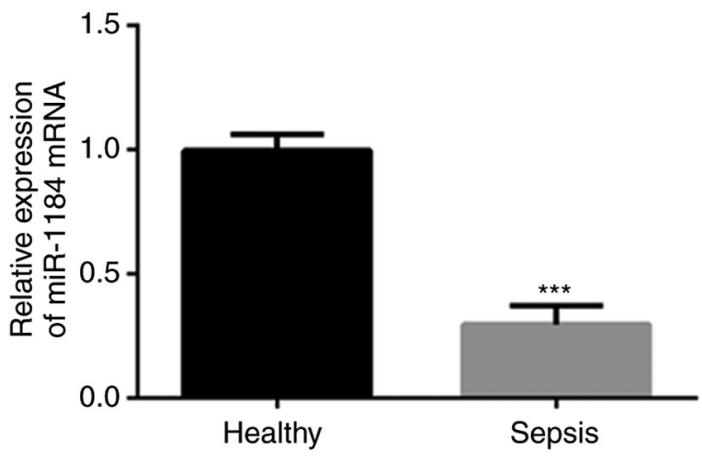

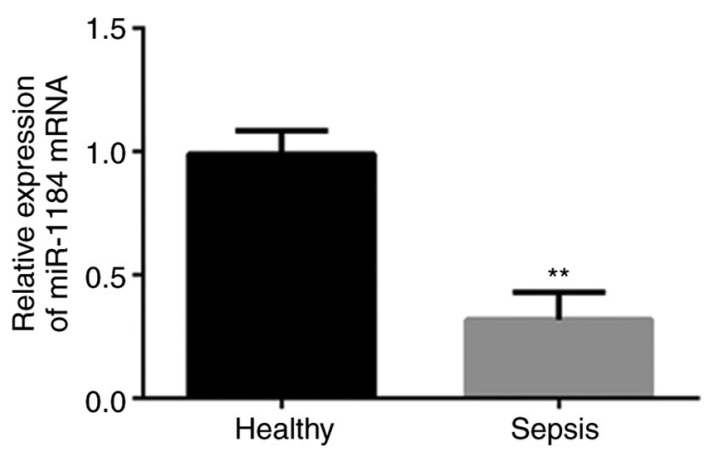

Figure 1. miR-1184 expression is significantly decreased in clinical specimens from children with sepsis. Reverse transcription-quantitative PCR was carried out to detect the expression level of miR-1184 in (A) blood mononuclear cells and (B) serum samples. Data are expressed as mean $\pm \mathrm{SD}(\mathrm{n}=30)$. ${ }^{* *} \mathrm{P}<0.01$, ${ }^{* * *} \mathrm{P}<0.001$ vs. healthy controls. miR, microRNA.

TargetScan analysis. The binding sites between miR-1184 and TRADD were predicted using TargetScan bioinformatics software (version 7.2; http://www.targetscan.org/vert_72/).

Luciferase reporter assay. An association between miR-1184 and TRADD was predicted using TargetScan software. A luciferase reporter assay was then performed to verify the putative binding sites. The DNA sequence of the TRADD 3'-untranslated region (3'-UTR) containing wild-type or mutant target sites for miR-1184 was subcloned and inserted into a pGL3-Control Vector (Promega Corporation) to create wild-type TRADD (TRADD-WT) or mutated-TRADD (TRADD-MUT) reporter vectors, respectively. Subsequently, THP-1 cells were co-transfected with a luciferase reporter vector and either miR-1184 mimic or miR-NC for $48 \mathrm{~h}$ using Lipofectamine ${ }^{\circledR} 2000$ (Invitrogen; Thermo Fisher Scientific, Inc.). Relative luciferase activities were detected using a Dual-Luciferase Reporter Assay kit (Promega Corporation), which was compared with Renilla luciferase activity according to the manufacturer's instructions.

Western blot analysis. Cells were inoculated in a 96-well plate at a concentration of $1 \times 10^{3} /$ well. The cells were treated with LPS, as previously described, and then total protein was extracted from the cells using RIPA buffer (Auragene; Hunan Aijia Biotechnology Co., Ltd.). Protein quantities were determined using a BCA protein assay kit (Bio-Rad Laboratories, Inc.). Samples containing $40 \mu \mathrm{g}$ protein/lane were separated by $10 \%$ SDS-PAGE and transferred onto PVDF membranes (EMD Millipore). Following blocking with 5\% non-fat milk for $1 \mathrm{~h}$ at room temperature, the membranes were incubated with primary antibodies targeting TRADD $(1: 1,000$; cat. no. ab110644; Abcam), p65 (1:1,000; cat. no. ab16502; Abcam), phosphorylated (p)-p65 (1:1,000; cat. no. ab183559; Abcam) and GAPDH (1:1,000; cat. no. ab9485; Abcam) overnight at $4^{\circ} \mathrm{C}$. Following washing with $0.1 \%$ PBS-Tween-20 four times, the membranes were incubated with horseradish peroxidase-conjugated secondary antibody (1:5,000; ab150113; Abcam) for $1 \mathrm{~h}$ at room temperature. Finally, the bands were visualized using an ECL detection system (Beyotime Institute of Biotechnology) and Image J software (version 146; National Institutes of Health) was used to analyze the fold-changes of protein levels.

Statistical analysis. SPSS version 20.0 (IBM Corp.) and GraphPad Prism 6.0 (GraphPad Software, Inc.) were used to analyze the data. All results are presented as the mean \pm SD from at least three independent experiments. The differences between and among groups were evaluated using Student's t-test or one-way ANOVA followed by Tukey's post hoc test, respectively. $\mathrm{P}<0.05$ was considered to indicate a statistically significant difference.

\section{Results}

miR-1184 expression is downregulated in clinical samples from children with sepsis. To assess the expression levels of miR-1184 in the blood of children with sepsis, RT-qPCR was performed. As shown in Fig. 1A, compared with healthy controls, miR-1184 expression was significantly decreased in the blood mononuclear cells of patients with sepsis. Similar results were observed for the detection of miR-1184 expression 
A

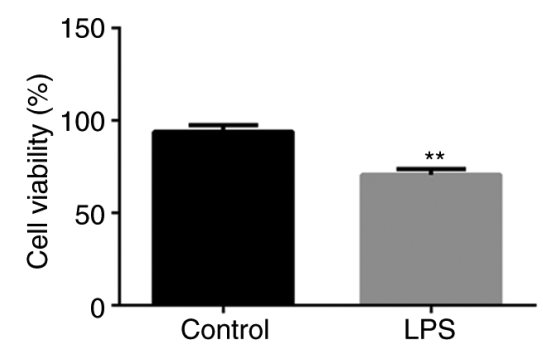

B

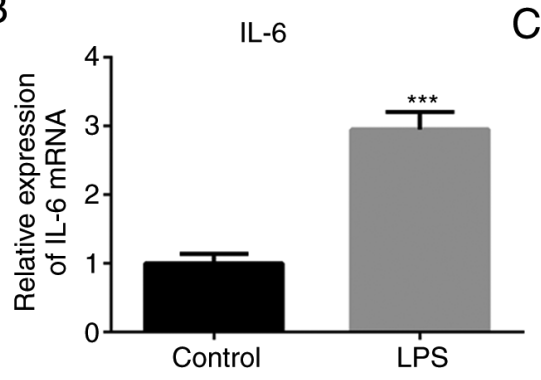

C

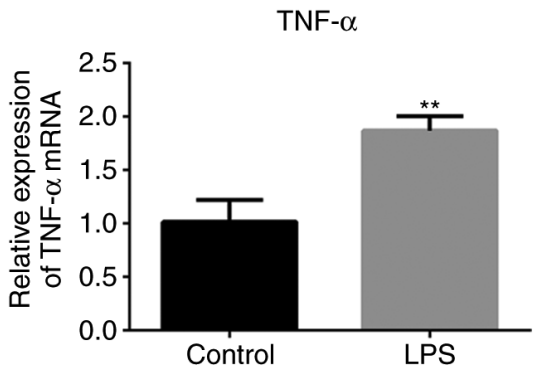

D

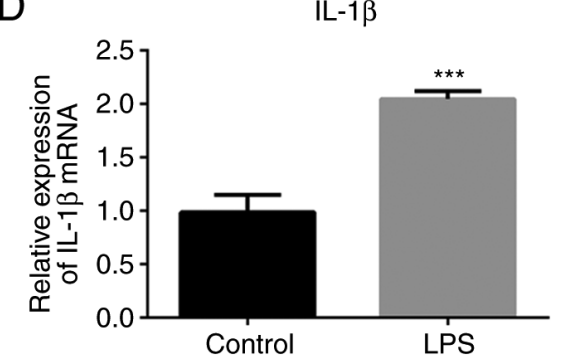

$E$

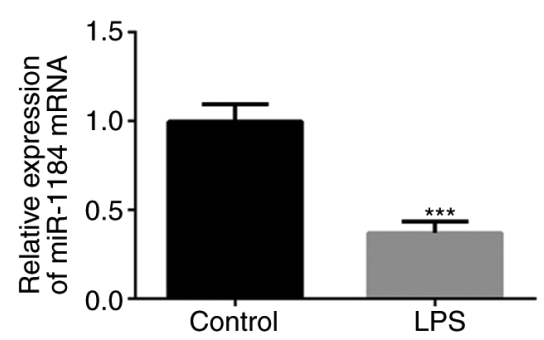

Figure 2. Effects of LPS on the viability, miR-1184 expression and inflammatory factors of THP-1 cells. (A) A Cell Counting Kit- 8 assay was used to detect cell viability. The expression levels of (B) IL-6, (C) TNF- $\alpha$ and (D) IL-1 $\beta$ mRNAs were significantly promoted by stimulation with LPS. (E) The mRNA expression of miR-1184 was reduced after LPS treatment. Data are expressed as mean $\pm \mathrm{SD}(\mathrm{n}=5){ }^{* *} \mathrm{P}<0.01,{ }^{* * *} \mathrm{P}<0.001$ vs. control. miR, microRNA; LPS, lipopolysaccharide; IL, interleukin; TNF, tumor necrosis factor.

in the serum (Fig. 1B). The data indicate that miR-1184 may be associated with the occurrence of sepsis in children.

Levels of miR-1184 and inflammatory factors are increased and decreased, respectively, in THP-1 cells under LPS treatment. To assess whether the in vitro model of sepsis was successfully established, a CCK-8 assay was conducted to detect the cell viability. LPS was shown to inhibit cell viability compared with that of the control group without LPS stimulation (Fig. 2A). The levels of representative inflammatory mediators were then detected using RT-qPCR. As presented in Fig. 2B-D, the expression levels of IL-6, TNF- $\alpha$ and IL-1 $\beta$ were significantly increased in the LPS-induced THP-1 cells compared with cells without LPS stimulation. In addition, miR-1184 expression was significantly decreased in the LPS-induced cells (Fig. 2E), which is consistent with the results shown in Fig. 1.

miR-1184 overexpression inhibits the production of inflammatory cytokines and apoptosis induced by LPS. To investigate the role of miR-1184 in LPS-induced THP-1 cells, miR-1184 was overexpressed by transfection with miR-1184 mimic. RT-qPCR results showed that miR-1184 expression was significantly increased following transfection with miR-1184 mimic compared with miR-NC under conditions of LPS stimulation (Fig. 3A). The LPS-induced levels of IL- 6 , TNF- $\alpha$ and IL- $1 \beta$ were decreased by transfection with miR-1184 mimic compared with those in the negative control (LPS + miR-NC) group (Fig. 3B-D). Furthermore, cell apoptosis rates in the LPS + miR-NC group were significantly increased compared with those in the control + miR-NC group, while the miR-1184 mimic decreased the LPS-induced cell apoptosis (Fig. 3E). These results indicate that the overexpression of miR-1184 plays a suppressive role in the LPS-induced inflammation and apoptosis of THP-1 cells.

Direct interaction between $\mathrm{miR}-1184$ and $T R A D D$. To verify the relationship between miR-1184 and TRADD, wild-type and mutant TRADD 3'-UTR sequences containing predicted miR-1184 binding sites were transfected into THP-1 cells (Fig. 4A). The results from the dual-luciferase reporter assay revealed that the luciferase activity significantly declined following co-transfection with TRADD (WT) and miR-1184 mimic, whereas no obvious effect of miR-1184 mimic was observed on the mutant TRADD 3'-UTR (Fig. 4B). Additionally, the mRNA expression level of TRADD was reduced by the upregulation of miR-1184 (Fig. 4C). Furthermore, LPS stimulation significantly induced TRADD expression in THP-1 cells (Fig. 4D). These results suggest that TRADD is a direct target of miR-1184.

miR-1184 attenuates inflammatory responses and apoptosis of THP-1 cells induced by LPS by targeting TRADD. To assess the role of TRADD in sepsis, TRADD was overexpressed in THP-1 cells. Western blotting results showed that the protein levels of TRADD were significantly increased following transfection with TRADD overexpression vector, and the increased levels were reduced by co-transfection with miR-1184 mimic (Fig. 5A). The effects of TRADD overexpression on the levels of inflammatory factors were then measured. As shown in Fig. 5B, compared with LPS-induced cells transfected with miR-1184 mimic, the expression levels of IL-6, TNF- $\alpha$ and IL-1 $\beta$ were significantly increased in cells following co-transfection with miR-1184 mimic and pcDNA-TRADD. In addition, the overexpression of TRADD attenuated the suppression of cell apoptosis induced by the miR-1184 mimic (Fig. 5C). The results indicate that TRADD reverses the effects 

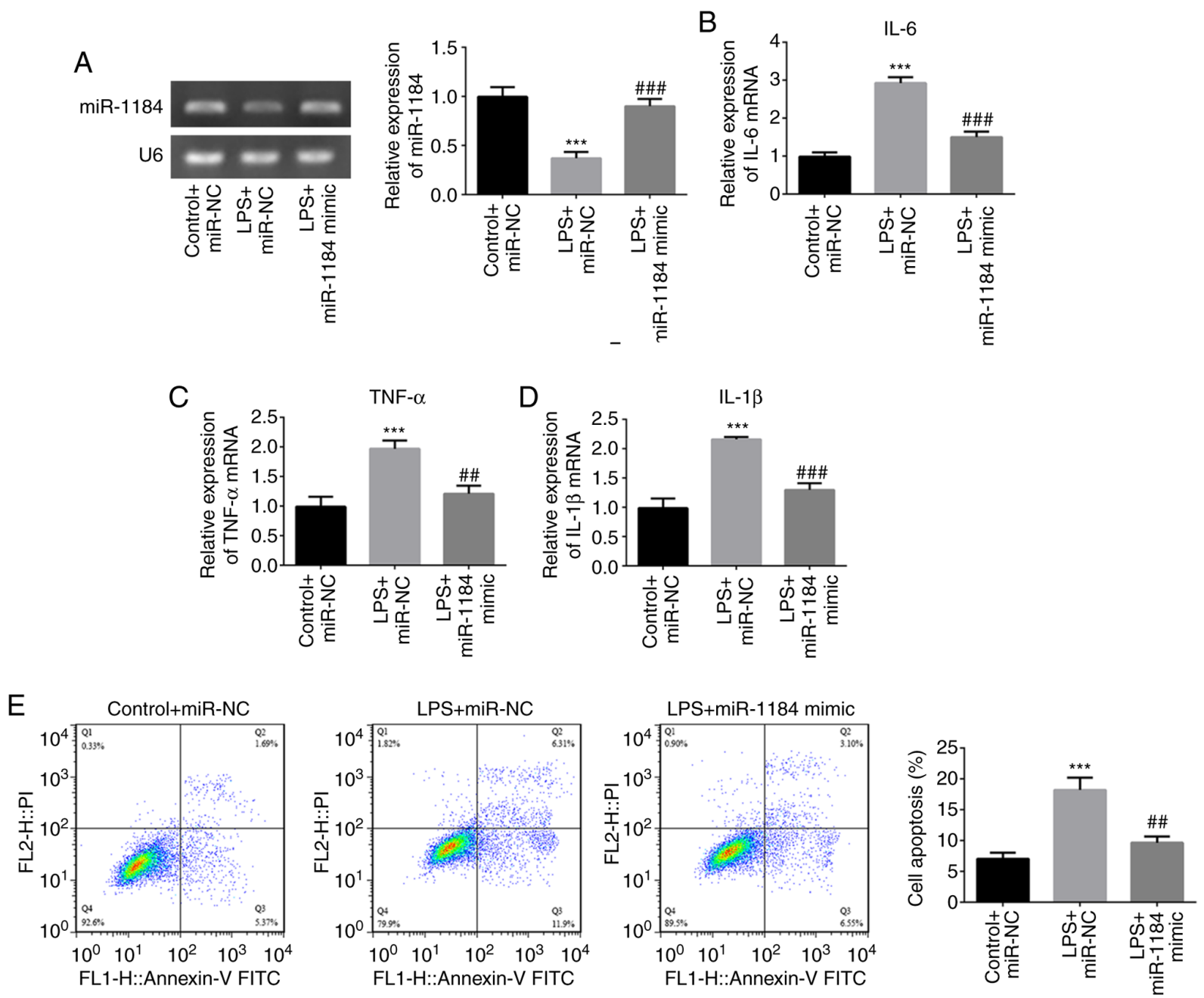

Figure 3. Overexpression of miR-1184 suppresses the LPS-induced expression of inflammatory cytokines and cell apoptosis. (A) miR-1184 mimic increased the expression of miR-1184 in LPS-stimulated THP-1 cells. The expression levels of (B) IL-6, (C) TNF- $\alpha$ and (D) IL-1 $\beta$ mRNAs in LPS-stimulated THP-1 cells were significantly decreased after transfection with miR-1184 mimic. (E) LPS-stimulated THP-1 cell apoptosis was inhibited by miR-1184 overexpression. Data are expressed as mean $\pm \mathrm{SD}(\mathrm{n}=5) .{ }^{* * *} \mathrm{P}<0.001$ vs. control + miR-NC; ${ }^{\# \#} \mathrm{P}<0.01,{ }^{\# \# \#} \mathrm{P}<0.001$ vs. LPS + miR-NC. miR, microRNA; LPS, lipopolysaccharide; $\mathrm{NC}$, negative control; IL, interleukin; TNF, tumor necrosis factor.

\section{A tradd (WT) : : 5' Gacuucauccugggugcugcaga $3^{\prime}$ miR-1184: : $3^{\prime}$ CCUUCGGUAGUUCAG - CGACGUCC 5'$$
\text { IIII }|||||| \mid
$$$$
\text { TRADD (MUT) : : } 5^{\prime} \quad \text { GACUUCAUCCUGGGUAUGUACUA } 3^{\prime}
$$
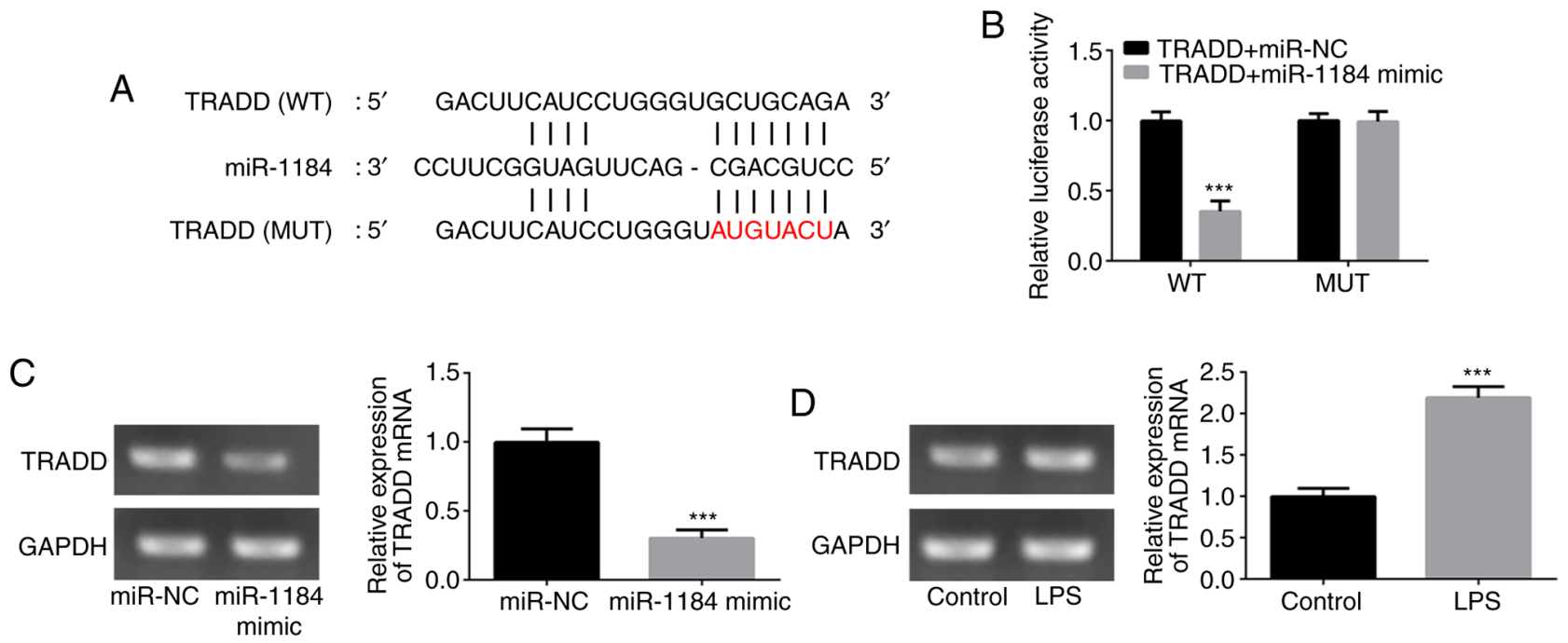

Figure 4. miR-1184 directly targets TRADD. (A) Predicted binding sites between miR-1184 and the TRADD 3'-untranslated region. (B) Relative luciferase activity measured after co-transfection with TRADD (WT)/(MUT) and miR-1184 mimic/miR-NC. (C) miR-1184 overexpression inhibited TRADD expression in THP-1 cells. (D) TRADD expression was increased after LPS stimulation compared with the unstimulated control. Data are expressed as mean \pm SD $(\mathrm{n}=3) .{ }^{* * *} \mathrm{P}<0.001$ vs. respective control. miR, microRNA; TRADD, TNF receptor type 1-associated DEATH domain protein; WT, wild type; MUT, mutant; $\mathrm{NC}$, negative control; LPS, lipopolysaccharide. 
A

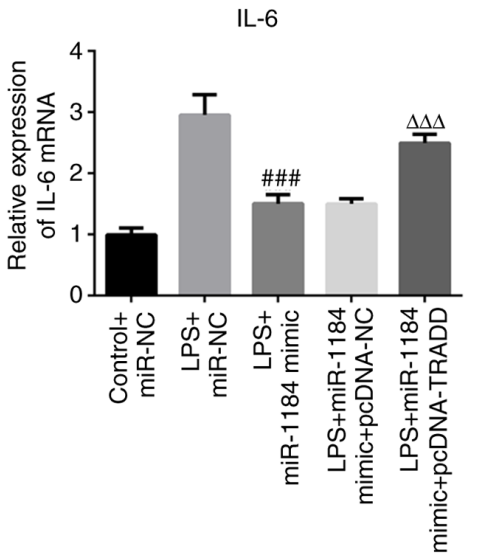

C

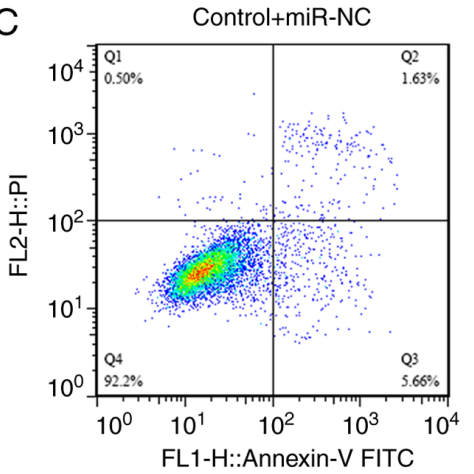

LPS+miR-1184 mimic + pcDNA-NC

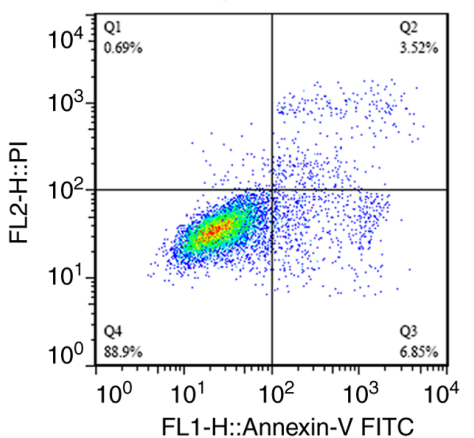

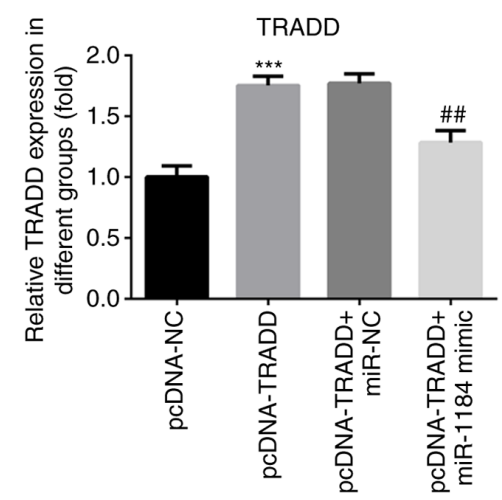

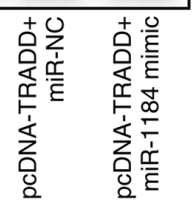

TNF- $\alpha$
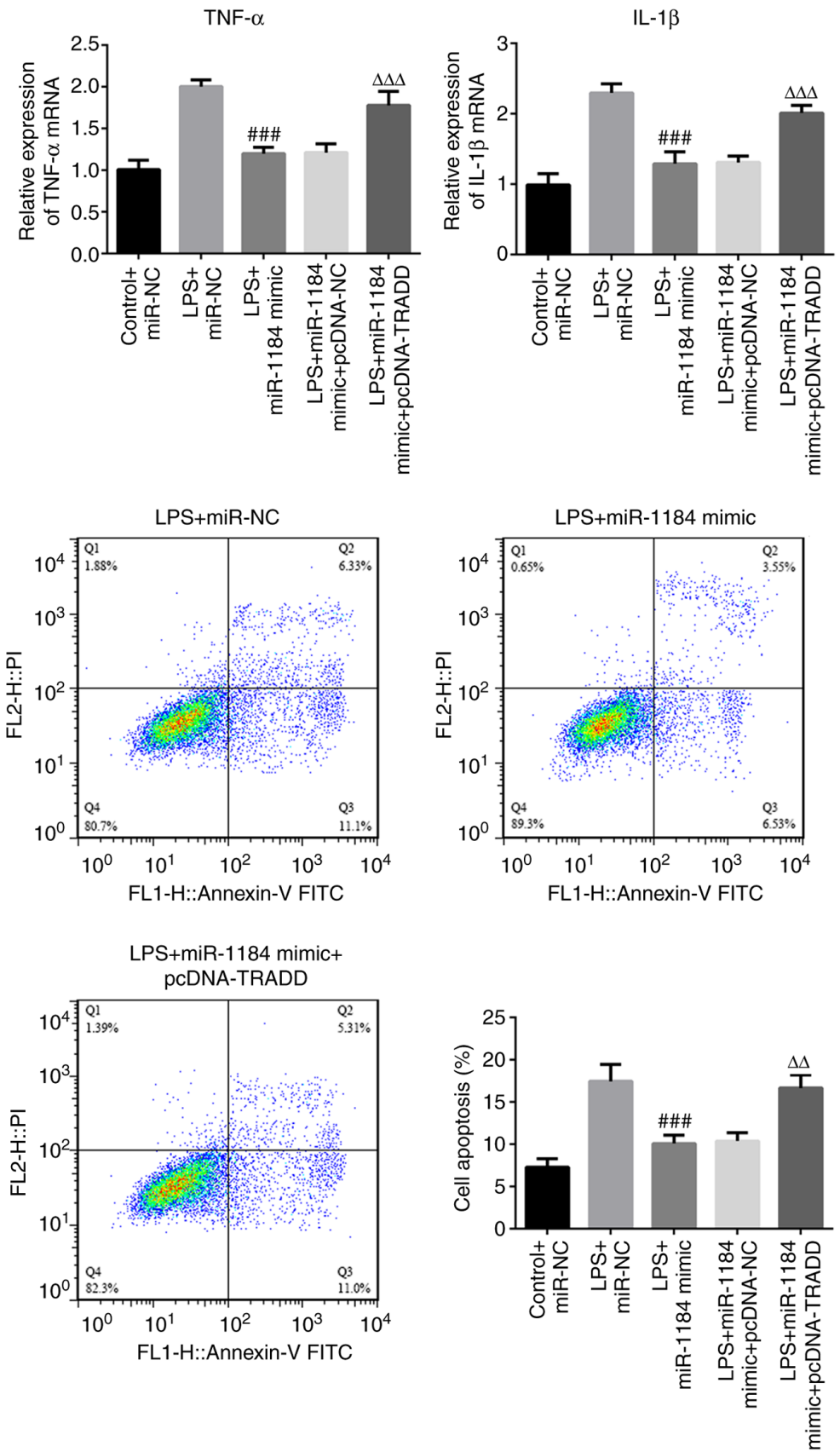

Figure 5. Effects of TRADD overexpression on the LPS-induced inflammatory responses and apoptosis of THP-1 cells. (A) Protein expression of TRADD was assessed after transfection with TRADD overexpression vector with or without miR-1184 mimic/miR-NC. ${ }^{* * *} \mathrm{P}<0.001$ vs. pcDNA-NC; ${ }^{\#} \mathrm{P}<0.01$ vs. pcDNA-TRADD + miR-NC. (B) The expression levels of IL-6, TNF- $\alpha$ and IL-1 $\beta$ in the miR-1184 mimic-transfected cells were significantly elevated after transfection with TRADD overexpression vector. (C) Flow cytometry was performed to estimate cell apoptosis in THP-1 cells transfected with miR-1184

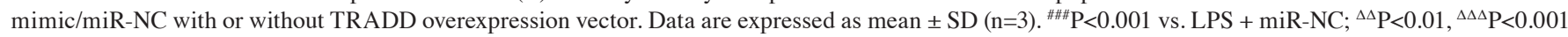
vs. LPS + miR-1184 mimic+ pcDNA-NC. TRADD, TNF receptor type 1-associated DEATH domain protein; LPS, lipopolysaccharide; miR, microRNA; $\mathrm{NC}$, negative control; IL, interleukin; TNF, tumor necrosis factor. 

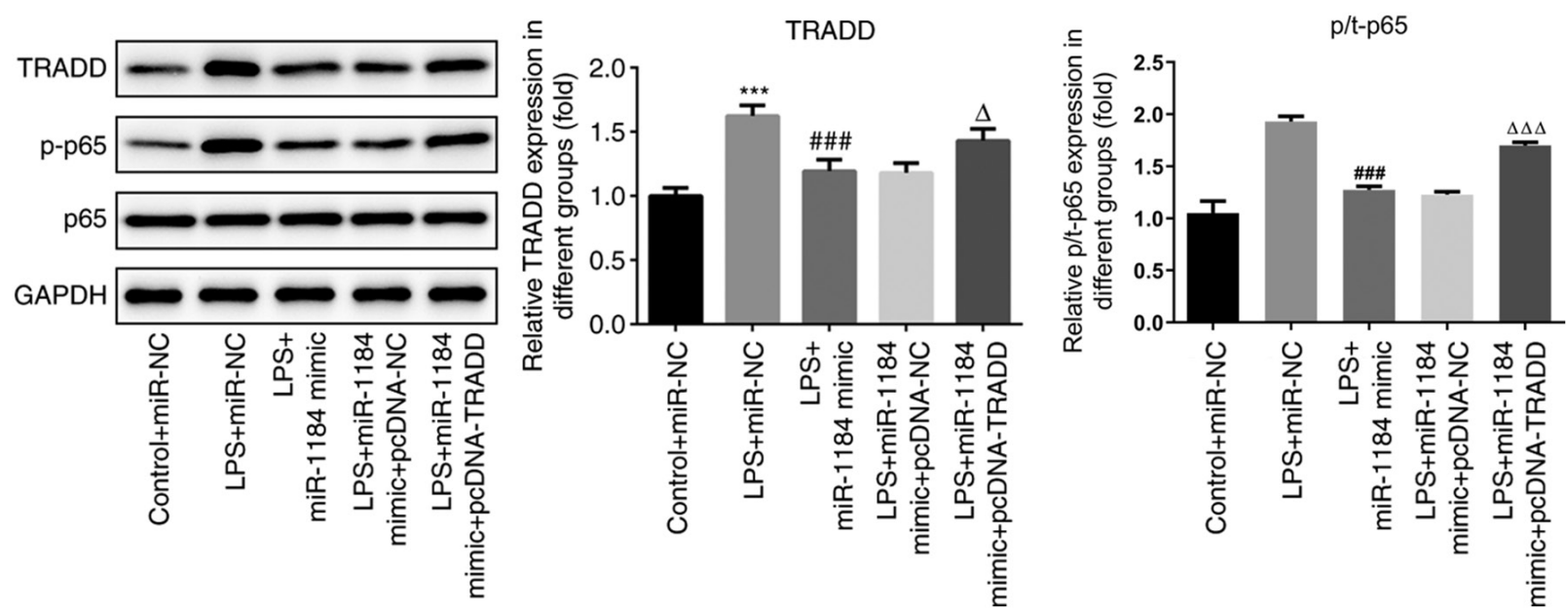

Figure 6. NF-kB signaling participates in the regulatory effect of miR-1184/TRADD in LPS-induced THP-1 cells. The protein levels of TRADD, p65 and p-p65 were detected by western blot analysis under LPS treatment after transfection with miR-1184 mimic/miR-NC with or without pcDNA-TRADD/pcDNA-NC. Data are expressed as mean $\pm \mathrm{SD}(\mathrm{n}=3) .{ }^{* * *} \mathrm{P}<0.001$ vs. control + miR-NC; ${ }^{\# \# *} \mathrm{P}<0.001$ vs. LPS + miR-NC; ${ }^{\Delta} \mathrm{P}<0.05,{ }^{\Delta \Delta \Delta} \mathrm{P}<0.01$ vs. LPS + miR-1184 mimic + pcDNA-NC. miR, microRNA; TRADD, TNF receptor type 1-associated DEATH domain protein; LPS, lipopolysaccharide; NC, negative control; p, phosphorylated.

of miR-1184 on the inflammatory response and apoptosis of LPS-induced THP-1 cells.

miR-1184/TRADD exerts biological functions in LPS-induced $T H P-1$ cells via regulation of $N F-\kappa B$ signaling. To further study the underlying mechanism of miR-1184 in sepsis, the levels of proteins associated with the NF- $\mathrm{kB}$ signaling pathway were detected. As shown in Fig. 6, the protein levels of TRADD and p-p65 were significantly increased by LPS stimulation. miR-1184 mimic repressed the LPS-induced protein expression of TRADD and p-p65, while TRADD overexpression reversed these effects. The data indicate that miR-1184 and TRADD exert biological functions in LPS-induced THP-1 cells via the regulation of NF- $\mathrm{kB}$ signaling.

\section{Discussion}

Sepsis is a blood infection mainly of bacterial, viral or fungal (yeast) origin that leads to substantial morbidity and mortality worldwide (19-21). The present study compared the expression of miR-1184 between children with sepsis and healthy children and found that miR-1184 expression was significantly decreased in the mononuclear cells and serum from the blood of the children with sepsis. A previous study revealed that the expression of numerous miRNAs were altered in neonatal patients with sepsis compared with uninfected neonates, among which miR-1184 was reported to be significantly reduced in the blood samples of the neonates with sepsis (15). To further understand the role of miR-1184 in childhood sepsis, an in vitro sepsis model was established in the present study by the LPS treatment of THP-1 human monocytic leukemia cells. THP-1 cells were treated with $1 \mu \mathrm{g} / \mathrm{ml}$ LPS for $24 \mathrm{~h}$ to simulate a septic environment $(16,17)$. Following construction of the in vitro sepsis model, the expression levels of IL-6, TNF- $\alpha$ and IL- $1 \beta$ were detected. miR-1184 expression in THP-1 cells was shown to decline under LPS stimulation. Following the overexpression of miR-1184, the production of the pro-inflammatory cytokines IL- 6 , TNF- $\alpha$ and IL- $1 \beta$ was inhibited and cell apoptosis decreased in LPS-induced cells. In the present study, a binding site between miR-1184 and TRADD was predicted using bioinformatics analysis, and the predicted binding between miR-1184 and TRADD was verified through a dual-luciferase reporter assay. Following the overexpression of miR-1184, the expression of TRADD in the THP-1 cells significantly decreased, indicating that miR-1184 can target and regulate TRADD. In addition, the overexpression of TRADD was found to attenuate the inhibitory effect of miR-1184 overexpression on apoptosis. Therefore, it may be concluded that miR-1184 directly regulates cell proliferation and other functions by interacting with TRADD. Moreover, western blotting results indicated that the NF- $\mathrm{KB}$ signaling pathway was inactivated in THP-1 cells after transfection with miR-1184 mimic.

miRNAs are small noncoding single-stranded RNA molecules that modulate gene expression and are involved in cellular physiological activities, including proliferation, invasion, metabolism and apoptosis (22-24). Alterations in miRNA expression play a crucial role in the regulation of the immune response (25). For example, Wu et al (26) reported that miR-23b expression was downregulated in LPS-induced vascular endothelial cells and miR-23b mimic inhibited the expression of inflammatory factors during sepsis. miR-1184 has been found to have biological functions in several diseases. For instance, Yang et al (27) revealed that miR-1184 was involved in the regulatory effect of circular RNA VANGL1 silencing on the progression of bladder cancer. In addition, changes in miR-1184 expression have been reported in breast cancer and prostatic benign hyperplasia $(28,29)$. In the present study, miR-1184 expression was observed to be reduced in the mononuclear cells and serum isolated from the blood samples of children with sepsis, as well as in LPS-induced THP-1 cells. Furthermore, overexpression of miR-1184 decreased the levels of the LPS-stimulated inflammatory cytokines TNF- $\alpha$, IL-1 $\beta$ and IL-6, and inhibited LPS-induced apoptosis in THP-1 cells. These results indicate that miR-1184 plays an inhibitory role in the pathophysiology of sepsis. 
Studies have demonstrated that miRNAs exert biological effects by binding to specific mRNA molecules and degrading mRNA (30). The present study showed that TRADD is a direct target of miR-1184. In addition, the overexpression of miR-1184 reduced TRADD expression, while the expression of TRADD was elevated by LPS stimulation. TRADD is a multifunctional protein that is important in TNF receptor 1 (TNFR1) signaling and other signaling pathways involved in immune responses (31). TRADD serves a role in the TNF- $\alpha$-induced pro-inflammatory response by interacting with TNFR1 and regulates the TLR signaling pathway by participating in the formation of complexes with TLR4 under LPS induction (31). Consistent with previous studies, the present results showed that TRADD promoted LPS-induced inflammatory cytokine production and cell apoptosis as it attenuated the inhibitory effects of miR-1184 overexpression on inflammatory responses and apoptosis in sepsis.

A previous study demonstrated that the phosphorylation level of p65 in TRADD-deficient cells was reduced following LPS treatment (32). TRADD serves a vital role in TIR domain-containing adapter-inducing interferon- $\beta$ (TRIF) signaling by participating in TRIF-dependent NF- $\kappa \mathrm{B}$ activation and regulating the production of inflammatory cytokines (33). Thus, we hypothesized that miR-1184 may modulate $\mathrm{NF}-\kappa \mathrm{B}$ signaling by directly targeting TRADD. The present study observed that the phosphorylation of p65 was upregulated upon LPS stimulation. miR-1184 overexpression suppressed p65 phosphorylation and TRADD attenuated the effects of miR-1184. These findings suggest that NF- $\kappa$ B activation may be associated with the regulatory role of miR-1184 on TRADD expression.

In summary, the present study suggests that miR-1184 may exert a therapeutic role in sepsis by repressing inflammatory cytokine production and cell apoptosis. Bioinformatics analysis and mechanistic investigations revealed that miR-1184 bound to the 3'-UTR of TRADD and mediated NF- $\kappa$ B signaling in an in vitro model of sepsis. These findings may contribute to the understanding of the pathogenesis of sepsis and the development of efficient therapies for children with sepsis.

\section{Acknowledgements}

Not applicable.

\section{Funding}

This study was supported by Investigation and Analysis of Children with Sepsis in Guizhou Province (grant no. 20161001).

\section{Availability of data and materials}

The analyzed data sets generated during the present study are available from the corresponding author on reasonable request.

\section{Authors' contributions}

PL and JC designed the study, and drafted and revised the manuscript. RT searched the literature. HW analyzed the data. PL, RT, HW and XD performed the experiments. All authors read and approved the final manuscript.

\section{Ethics approval and consent to participate}

Experiments were approved by the Ethics Committee of Guiyang Maternal and Child Health Care Hospital and written informed consent was signed by the parents or guardians of all participants.

\section{Patient consent for publication}

Not applicable.

\section{Competing interests}

The authors declare that they have no competing interests.

\section{References}

1. Vincent JL, Opal SM, Marshall JC and Tracey KJ: Sepsis definitions: Time for change. Lancet 381: 774-775, 2013.

2. Angus DC, Linde-Zwirble WT, Lidicker J, Clermont G, Carcillo J and Pinsky MR: Epidemiology of severe sepsis in the United States: Analysis of incidence, outcome, and associated costs of care. Crit Care Med 29: 1303-1310, 2001.

3. Skrupky LP, Kerby PW and Hotchkiss RS: Advances in the management of sepsis and the understanding of key immunologic defects. Anesthesiology 115: 1349-1362, 2011.

4. Zilberberg MD, Shorr AF, Micek ST, Vazquez-Guillamet $C$ and Kollef MH: Multi-drug resistance, inappropriate initial antibiotic therapy and mortality in Gram-negative severe sepsis and septic shock: A retrospective cohort study. Crit Care 18: 596, 2014.

5. Martin GS, Mannino DM and Moss M: The effect of age on the development and outcome of adult sepsis. Crit Care Med 34: 15-21, 2006.

6. Cecconi M, Evans L, Levy M and Rhodes A: Sepsis and septic shock. Lancet 392: 75-87, 2018.

7. Plunkett A and Tong J: Sepsis in children. BMJ 350: h3017, 2015.

8. Martinot A, Leclerc F, Cremer R, Leteurtre S, Fourier C and Hue V: Sepsis in neonates and children: Definitions, epidemiology, and outcome. Pediatr Emerg Care 13: 277-281, 1997.

9. Ambros V: The functions of animal microRNAs. Nature 431: 350-355, 2004.

10. Bartel DP: MicroRNAs: Genomics, biogenesis, mechanism, and function. Cell 116: 281-297, 2004.

11. Ward LD and Kellis M: HaploReg v4: Systematic mining of putative causal variants, cell types, regulators and target genes for human complex traits and disease. Nucleic Acids Res 44: D877-D881, 2016.

12. Tufekci KU, Oner MG, Meuwissen RL and Genc S: The role of microRNAs in human diseases. Methods Mol Biol 1107: 33-50, 2014.

13. Wang X, Wang X, Liu X, Wang X, Xu J, Hou S, Zhang X and Ding Y: miR-15a/16 are upreuglated in the serum of neonatal sepsis patients and inhibit the LPS-induced inflammatory pathway. Int J Clin Exp Med 8: 5683-5690, 2015.

14. Sheng B, Zhao L, Zang X, Zhen J and Chen W: miR-375 ameliorates sepsis by downregulating miR-21 level via inhibiting JAK2-STAT3 signaling. Biomed Pharmacother 86: 254-261, 2017.

15. Chen J, Jiang S, Cao Y and Yang Y: Altered miRNAs expression profiles and modulation of immune response genes and proteins during neonatal sepsis. J Clin Immunol 34: 340-348, 2014.

16. Cheng Q, Tang L and Wang Y: Regulatory role of miRNA-26a in neonatal sepsis. Exp Ther Med 16: 4836-4842, 2018.

17. How CK, Hou SK, Shih HC, Huang MS, Chiou SH, Lee CH and Juan CC: Expression profile of MicroRNAs in gram-negative bacterial sepsis. Shock 43: 121-127, 2015.

18. Livak KJ and Schmittgen TD: Analysis of relative gene expression data using real-time quantitative PCR and the 2(-Delta Delta C(T)) method. Methods 25: 402-408, 2001.

19. Muller MC, Meijers JC, Vroom MB and Juffermans NP: Utility of thromboelastography and/or thromboelastometry in adults with sepsis: A systematic review. Crit Care 18: R30, 2014.

20. Hermans G and Van den Berghe G: Clinical review: Intensive care unit acquired weakness. Crit Care 19: 274, 2015. 
21. Pavlakou P, Liakopoulos V, Eleftheriadis T, Mitsis M and Dounousi E: Oxidative stress and acute kidney injury in critical illness: Pathophysiologic mechanisms-biomarkers-interventions, and future perspectives. Oxid Med Cell Longev 2017: 6193694, 2017.

22. Lenkala D, LaCroix B, Gamazon ER, Geeleher P, Im HK and Huang RS: The impact of microRNA expression on cellular proliferation. Hum Genet 133: 931-938, 2014

23. Dvinge H, Git A, Graf S, Salmon-Divon M, Curtis C, Sottoriva A Zhao Y, Hirst M, Armisen J, Miska EA, et al: The shaping and functional consequences of the microRNA landscape in breast cancer. Nature 497: 378-382, 2013.

24. Melton C, Judson RL and Blelloch R: Opposing microRNA families regulate self-renewal in mouse embryonic stem cells. Nature 463: 621-626, 2010.

25. Tsitsiou E and Lindsay MA: microRNAs and the immune response. Curr Opin Pharmacol 9: 514-520, 2009.

26. Wu M, Gu JT, Yi B, Tang ZZ and Tao GC: microRNA-23b regulates the expression of inflammatory factors in vascular endothelial cells during sepsis. Exp Ther Med 9: 1125-1132, 2015.

27. Yang D, Qian H, Fang Z, Xu A, Zhao S, Liu B and Li D: Silencing circular RNA VANGL1 inhibits progression of bladder cancer by regulating miR-1184/IGFBP2 axis. Cancer Med 9: 700-710, 2020.

28. Danza K, De Summa S, Pinto R, Pilato B, Palumbo O, Carella M, Popescu O, Digennaro M, Lacalamita R and Tommasi S: TGFbeta and miRNA regulation in familial and sporadic breast cancer. Oncotarget 8: 50715-50723, 2017.
29. Knyazev EN, Fomicheva KA, Mikhailenko DS, Nyushko KM, Samatov TR, Ya Alekseev B and Yu Shkurnikov M: Plasma levels of hsa-miR-619-5p and hsa-miR-1184 differ in prostatic benign hyperplasia and cancer. Bull Exp Biol Med 161: 108-111, 2016.

30. Smillie CL, Sirey T and Ponting CP: Complexities of posttranscriptional regulation and the modeling of ceRNA crosstalk. Crit Rev Biochem Mol Biol 53: 231-245, 2018.

31. Chen NJ, Chio IIC, Lin WJ, Duncan G, Chau H, Katz D, Huang HL, Pike KA, Hao Z, Su YW, et al: Beyond tumor necrosis factor receptor: TRADD signaling in toll-like receptors. Proc Natl Acad Sci USA 105: 12429-12434, 2008.

32. Wilson NS, Dixit V and Ashkenazi A: Death receptor signal transducers: Nodes of coordination in immune signaling networks. Nat Immunol 10: 348-355, 2009.

33. Shukla K, Sharma AK, Ward A, Will R, Hielscher T, Balwierz A Breunig C, Münstermann E, König R, Keklikoglou I and Wiemann S: MicroRNA-30c-2-3p negatively regulates NF-kB signaling and cell cycle progression through downregulation of TRADD and CCNE1 in breast cancer. Mol Oncol 9: 1106-1119, 2015. International (CC BY-NC-ND 4.0) License. 